designed furnace. The mirror will now be transported from the works in Ohio to Kitt Peak where the grinding and figuring of the surface to an accuracy of one millionth of an inch will be carried out.

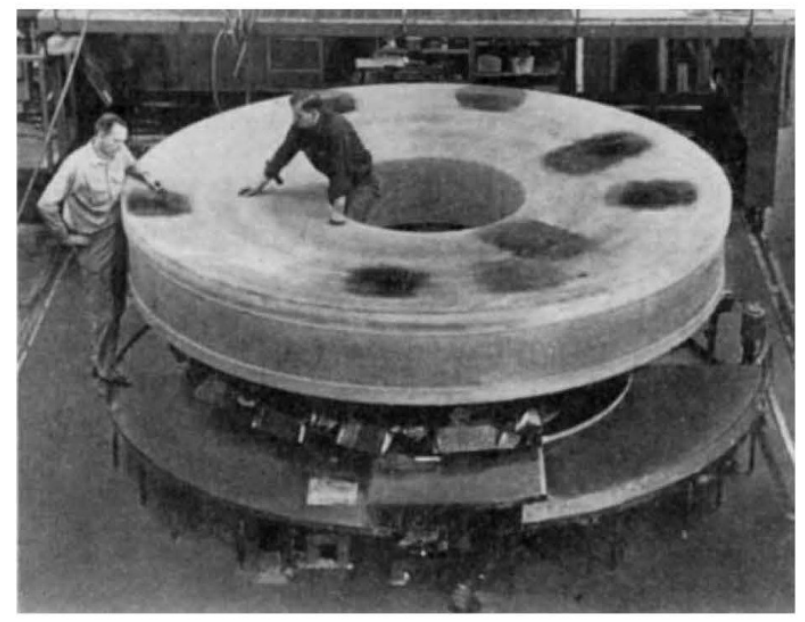

The world's largest quartz mirror blank, now ready for shaping and polishing for the Kitt Peak telescope.

When completed, the Kitt Peak telescope will be the largest telescope to use a quartz mirror instead of conventional glass. Quartz has the advantage that its expansion with temperature is one fifth that of ordinary glass, as well as being very hard and rigid. According to Mr R. A. Popp of $\mathrm{GE}(\mathrm{USA})$ it can be polished and finished faster than borosilicate glass. The Kitt Peak installation will be the first big telescope to use the Ritchey-Chrétien optical system. This form is not new, but is only now being generally adopted for astronomical telescopes. The Newtonian-Cassegraine system, used for almost all existing large telescopes, gives perfect axial imagery, but its inherent coma limits the useful field size, particularly at the prime focus. The RitcheyChrétien form gives a much larger coma-free secondary focus but the prime focus image has quite heavy spherical aberration. In either form the field of the prime focus can be extended by the use of afocal lens systems, but rather unexpectedly these give considerably wider useful fields on the Ritchey-Chrétien telescope.

The Optical Design Group at Imperial College under Dr C. G. Wynne has investigated field corrector systems for such telescopes as the 200 inch at Mount Palomar (Newtonian-Cassegraine), Macdonald (RitcheyChrétien) and Kitt Peak. The 108 inch Macdonald telescope, like that at Kitt Peak, is to use a quartz mirror and is at present under construction.

\section{Research into Blindness}

WANTED-one million pounds by the "Fight for Sight" campaign to provide an annual income of at least $£ 50,000$ for work undertaken at the Institute of Ophthalmology in London. Government financial aid to the institute is insufficient to cover the cost of the work; consequently, the institute must augment its income by private benefaction. The president and emeritus director of research, Sir Stewart Duke-Elder, was also the prime mover in establishing the institute in 1946, in association with Moorfields Medical School and as part of the University of London. All the teaching and part of the research costs are met by grants from the University of London and the institute has so far received $£ 419,345$ from other sources. This has enabled the setting up of two research fellowships for the study of glaucoma and a fellowship has also been initiated for the study of eye diseases and rtlated problems, particularly in children.

The research work in progress is extremely varied, but with more than five million blind children alive in the world today there can never be too many investigations into the causes and cures of blindness. Some types of blindness are caused by hereditary factors-retinitis pigmentosa or degeneration of the retina, for example. Work in this direction is conducted at the molecular level. Other types of retinal abnormalities are caused by environmental factors, as seen in premature babies who become blind when supplied with oxygen shortly after birth. Increased blood pressure or hypertension is another important factor determining defective sight. Under the direction of Professor N. Ashton, this condition is being induced experimentally in animals with a view to determining the cause of the defect. The use of lasers for bloodless surgery on the anterior part of the eye (iridectomy) is also being investigated at the institute and a laser slit lamp-really an ophthalmoscope incorporating a laserhas been developed there. Other projects include an investigation of visual pigments, laboratory diagnosis of eye diseases, electron microscopy and serial reconstruction of the retina as well as efforts to improve underwater vision.

The research teams are, however, hindered by lack of space. The construction of another floor at the institute is being debated but, until something definite is arranged, the number of staff will be limited and research programmes will take longer to complete.

\section{Generator for Surrey}

THE rewards of collaboration between universities and industry would appear to outweigh any possible loss of academic freedom. This is true, at least, for the University of Surrey. The Standard Telecommunication Laboratory has just presented the Electrical Engineering Department with a 2 million volt Van de Graaf generator. The generator is not new; it is, in fact, eleven years old and STL had no further use for it, but it seems admirably suited to the intentions of the department.

Professor D. R. Chick, of the Electrical Engineering Department, has a research team working in collaboration with the UKAEA on the implantation of heavy positively charged ions into semiconductors and metals. The generator will be used to implant impurities directly into silicon and germanium. It will also be used for X-ray and neutron studies of interest to the physics department and the radiation unit of the university. The machine will also be an invaluable teaching tool in the department's new M.Sc course on nuclear radiation and semiconductors which is to be run in collaboration with other departments of the university and the UKAEA, and which starts in October 1968.

This M.Se course will have its emphasis on the experimental and engineering techniques of radiation production and detection, and of diagnostic methods 